\title{
A Halo Substructure in Gaia Data Release 1
}

\author{
G. C. Myeong ${ }^{1 \star}$, N. W. Evans ${ }^{1}$, V. Belokurov ${ }^{1}$, S.E. Koposov ${ }^{1,2}$ \& J.L. Sanders ${ }^{1}$ \\ ${ }^{1}$ Institute of Astronomy, University of Cambridge, Madingley Road, Cambridge CB3 OHA. \\ ${ }^{2}$ McWilliams Center for Cosmology, Department of Physics, Carnegie Mellon University, 5000 Forbes Avenue, Pittsburgh, PA 15213, USA
}

version 27 April 2017.

\begin{abstract}
We identify a halo substructure in the Tycho Gaia Astrometric Solution (TGAS) dataset, cross-matched with the RAVE-on data release. After quality cuts, the stars with large radial action $\left(J_{R}>800 \mathrm{kms}^{-1} \mathrm{kpc}\right)$ are extracted. A subset of these stars is clustered in longitude and velocity and can be selected with further cuts. The 14 stars are centered on $(X, Y, Z) \approx(9.0,-1.0,-0.6) \mathrm{kpc}$ and form a coherently moving structure in the halo with median $\left(v_{R}, v_{\phi}, v_{z}\right)=(167.33,0.86,-94.85) \mathrm{kms}^{-1}$. They are all metal-poor giants with median $[\mathrm{Fe} / \mathrm{H}]=-0.83$. To guard against the effects of distance errors, we compute spectrophotometric distances for the 8 out of 14 stars where this is possible. We find that 6 of the stars are still comoving. These 6 stars also have a much tighter $[\mathrm{Fe} / \mathrm{H}]$ distribution $\sim-0.7$ with one exception $([\mathrm{Fe} / \mathrm{H}]=-2.12)$. We conclude that the existence of the comoving cluster is stable against changes in distance estimation and conjecture that this is the dissolving remnant of a long-ago accreted globular cluster.
\end{abstract}

Key words: galaxies: kinematics and dynamics - galaxies: structure

\section{INTRODUCTION}

Over the last decade, many streams have been identified in the stellar halo of the Milky Way, usually as overdensities of mainsequence turn-off stars in resolved star maps from wide area photometric surveys (see e.g., Belokurov et al. 2006; Grillmair 2009; Newberg \& Carlin 2015). An alternative method is to identify substructure kinematically as samples of stars with similar chemistry moving in a distinct and coherent way. Though less widely used, this has had some striking successes, including the famous identification of the Sagittarius dwarf (Ibata et al. 1994), the halo stream found by Helmi et al. (1999) in Hipparcos data, and the globular cluster streams found by Smith et al. (2009) in Sloan Digital Survey Stripe 82 data.

The first data releases (DR1) of the Gaia satellite (Gaia Collaboration et al. 2016a,b) provides us with a new vista of the Solar neighbourhood. The primary astrometric catalogue in Gaia DR1 is TGAS (Tycho Gaia Astrometric solution), which uses data from Tycho-2 (Høg et al. 2000) to provide a 30 year baseline for astrometric calculations. It has 2057050 entries. When cross-matched with RAVE-on (Casey et al. 2016; Kunder et al. 2017), this gives a catalogue of 180929 stars with full six-dimensional phase space information, as well as associated stellar spectral quantities. TGAS cross-matches are also possible with LAMOST (Luo et al. 2015) and APOGEE (Anders et al. 2014), though they are somewhat smaller in size with 78579 and 12061 entries respectively. Not all the entries in the three cross-matched catalogues are distinct. When

^ E-mail: gm564,nwe,vasily,koposov,jls@ast.cam.ac.uk

\begin{tabular}{ccc}
\hline \hline RAVE ID & RA & DEC \\
\hline J063314.9-284345 & 98.312 & -28.729 \\
J062653.3-355032 & 96.722 & -35.842 \\
J052906.3-240844 & 82.276 & -24.145 \\
J050209.4-235127 & 75.539 & -23.858 \\
J050519.6-264950 & 76.332 & -26.831 \\
\hline
\end{tabular}

Table 1. RAVE identifiers, as well as right ascension and declination, for the 5 confirmed members.

stars overlap in the surveys, we take the data with the smallest relative error in radial velocity. This yields a final master catalogue of 268588 stars where the RAVE-on contribution is 180454 stars. The master catalogue is a natural arena in which to search for kinematic substructure.

This Letter identifies a group of stars moving on strongly radial orbits in the TGAS cross-matched master catalogue. The stars have similar metallicities, and the simplest explanation of their unusual kinematics is that they are the residue of an ancient halo structure. Section 2 describes our treatment of the data and extraction of the substructure stars. Section 3 discusses the selected stars, together with estimates for the age and future evolution of the substructure. 

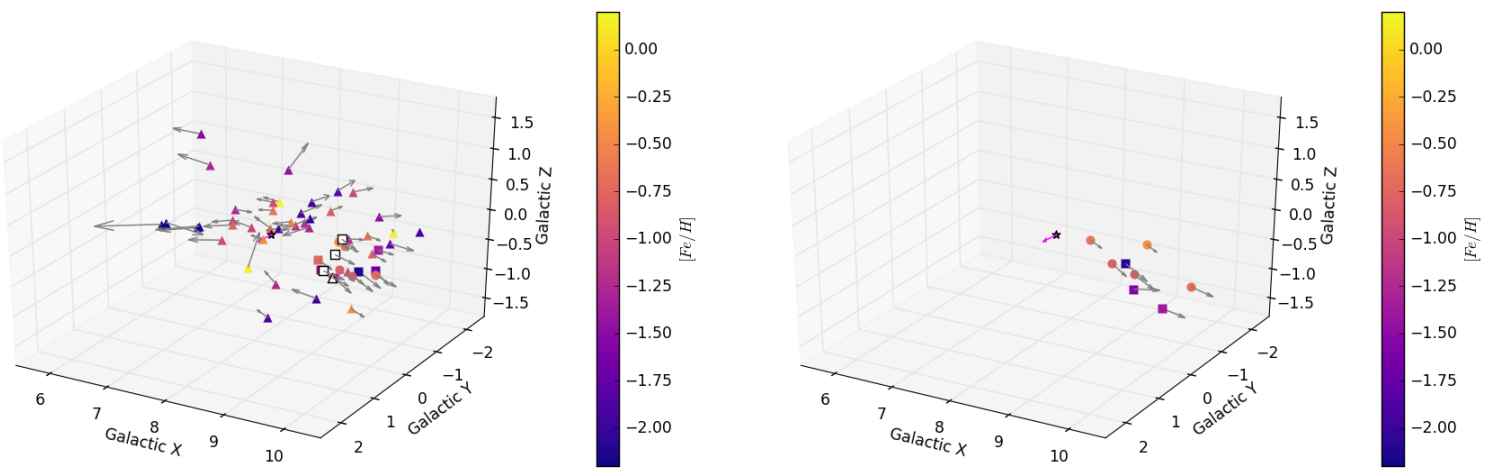

Figure 1. Left: The spatial structure of the 56 stars satisfying the quality cuts and with $J_{R}>800 \mathrm{kms}^{-1} \mathrm{kpc}$. Stars are colour-coded according to metallicity if known, whilst the arrow indicates the magnitude and direction of the spatial velocity. There is a clear clustering of stars at $(X, Y, Z) \approx(9.0,-1.0,-0.6)$ $\mathrm{kpc}$, which have radial motion dominating their total velocity. The 14 stars that may belong to the comoving cluster are shown as circles or squares, while the remainder are shown as triangles. Right: Using spectrophotometric distances, the 14 candidate members are re-examined. 5 stars (circles) are retained as confirmed members as both their distances and metallicities are similar. Of the remaining 9 stars, 6 do not have spectrophotometric distances. However, 2 are not comoving according to the new distances, whilst 1 is comoving but has markedly different metallicity. These 3 objects are marked with squares. Finally, the Sun is marked on both plots as a star.
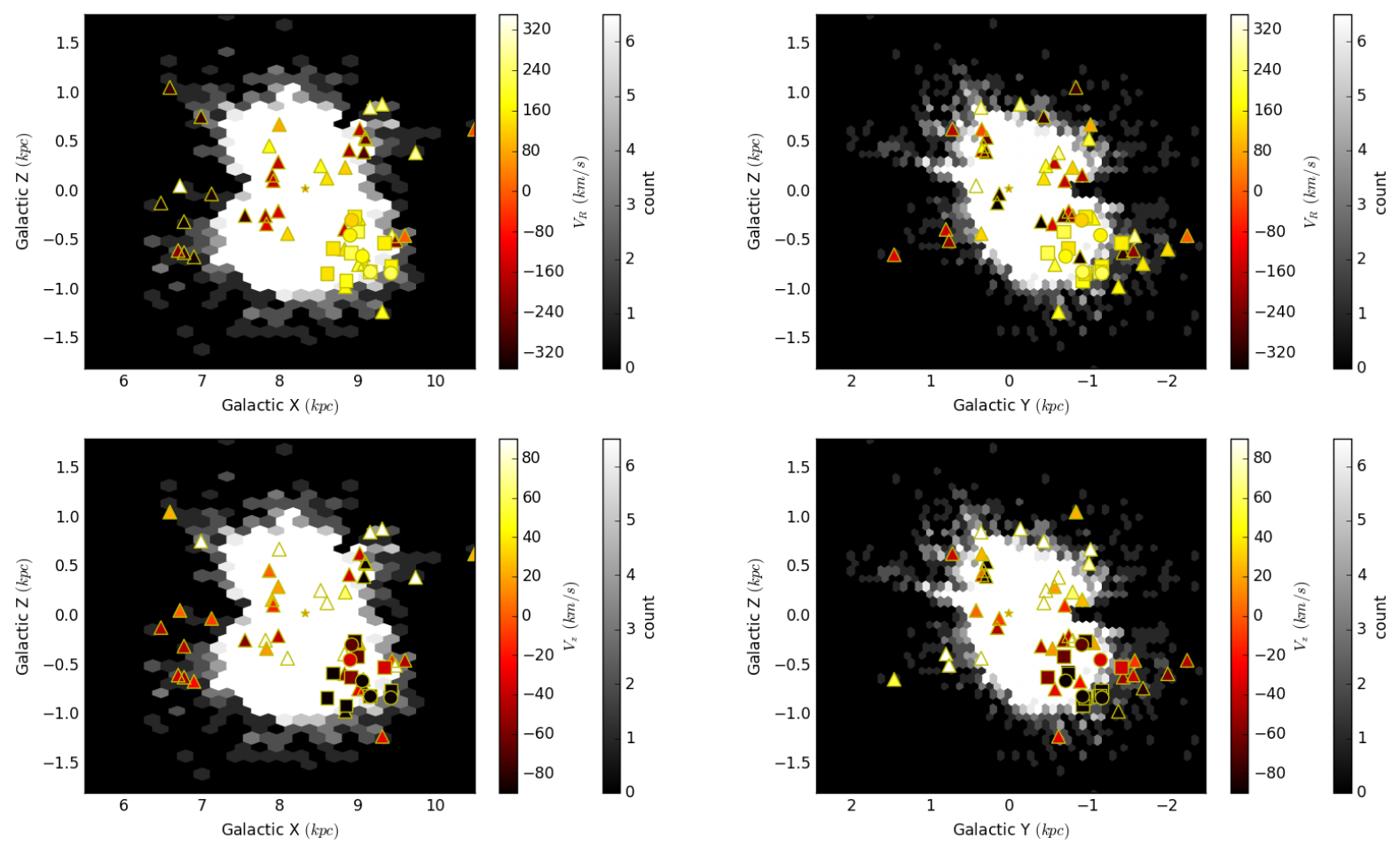

Figure 2. Locations of the 56 high-quality stars with $J_{R}>800 \mathrm{kms}^{-1} \mathrm{kpc}$ in the Galactic $(X, Y)$ and $(Y, Z)$ planes using the Astraatmadja \& Bailer-Jones (2016) distances. Circles ( 5 confirmed members) and squares (9 possibles) show stars associated with the comoving clump, whilst triangles show the rejected stars. Symbols are coloured according to the radial velocity (upper panels) or vertical velocity (lower panels). The colours of the symbols show that the stars of interest have quite similar velocitiues, both vertical and radial.

\section{EXTRACTION OF THE MEMBER STARS}

First, the proper motions are converted to velocities using the unbiased, inferred distance estimates of Astraatmadja \& Bailer-Jones (2016). To ensure a high quality sample, we impose a cut that the error in the radial velocity $\epsilon_{\mathrm{RV}}<10 \mathrm{kms}^{-1}$, the error in the total velocity $\epsilon_{\mathrm{Vtot}}<25 \mathrm{kms}^{-1}$ and the relative error in the total velocity is $<10 \%$. This reduces the sample to 73268 stars $(66891$ using the RAVE-on measurements, the remainder from LAMOST and
APOGEE). We now use the Galactic potential MWPotential2014 in Bovy (2015) to compute the radial and azimuthal actions $\left(J_{R}\right.$ and $J_{\phi}$, using the adiabatic approximation e.g. Binney 2010). The bulk of the stars are moving on nearly circular orbits in the thin disk. Nonetheless, there are extensions of stars in the high action regime, which are predominantly metal-poor and are moving on eccentric orbits.

We retain only stars with a radial action $J_{R}>800 \mathrm{kms}^{-1} \mathrm{kpc}$. 


\begin{tabular}{|c|c|c|c|c|c|c|c|c|c|}
\hline & {$[\mathrm{Fe} / \mathrm{H}]$} & $\log g$ & $\begin{array}{l}T_{\text {eff }} \\
(\mathrm{K})\end{array}$ & $\begin{array}{c}(X, Y, Z) \\
(\mathrm{kpc})\end{array}$ & $\begin{array}{c}\left(v_{R}, v_{\phi}, v_{z}\right) \\
\left(\mathrm{kms}^{-1}\right)\end{array}$ & $e$ & $\begin{array}{l}R_{\text {apo }} \\
(\mathrm{kpc})\end{array}$ & $\begin{array}{l}R_{\text {peri }} \\
(\mathrm{kpc})\end{array}$ & $\begin{array}{c}z_{\max } \\
(\mathrm{kpc})\end{array}$ \\
\hline Median & -0.83 & 1.93 & 4662 & $(9.00,-0.94,-0.62)$ & $(167.33,0.86,-94.85)$ & 0.940 & 12.79 & 0.40 & 3.50 \\
\hline Median Absolute Deviation & 0.28 & 0.74 & 355 & $(0.15,0.21,0.18)$ & $(21.47,10.18,30.67)$ & 0.017 & 1.55 & 0.11 & 1.45 \\
\hline \multirow[t]{2}{*}{ Median } & -0.70 & 2.11 & 4662 & $(9.06,-0.94,-0.66)$ & $(167.33,8.79,-94.86)$ & 0.948 & 12.31 & 0.40 & 3.50 \\
\hline & & & & $(9.13,-0.90,-0.53)$ & $(168.57,11.24,-72.96)$ & 0.947 & 12.02 & 0.29 & 4.50 \\
\hline \multirow[t]{2}{*}{ Median Absolute Deviation } & 0.10 & 0.27 & 268 & $(0.14,0.21,0.17)$ & $(44.35,8.11,27.22)$ & 0.011 & 1.98 & 0.13 & 1.69 \\
\hline & & & & $(0.21,0.37,0.25)$ & $(52.23,1.38,7.13)$ & 0.006 & 1.92 & 0.08 & 2.65 \\
\hline
\end{tabular}

Table 2. Upper: The medians and median absolute deviations in spectroscopic and orbital properties of all 14 possible stars in the comoving group. Lower: The same, but for the subset of 5 stars that are unambiguous members. In this case, the velocities and orbital parameters are given first using Astraatmadja \& Bailer-Jones (2016) distances, and then using spectrophotometric distances.

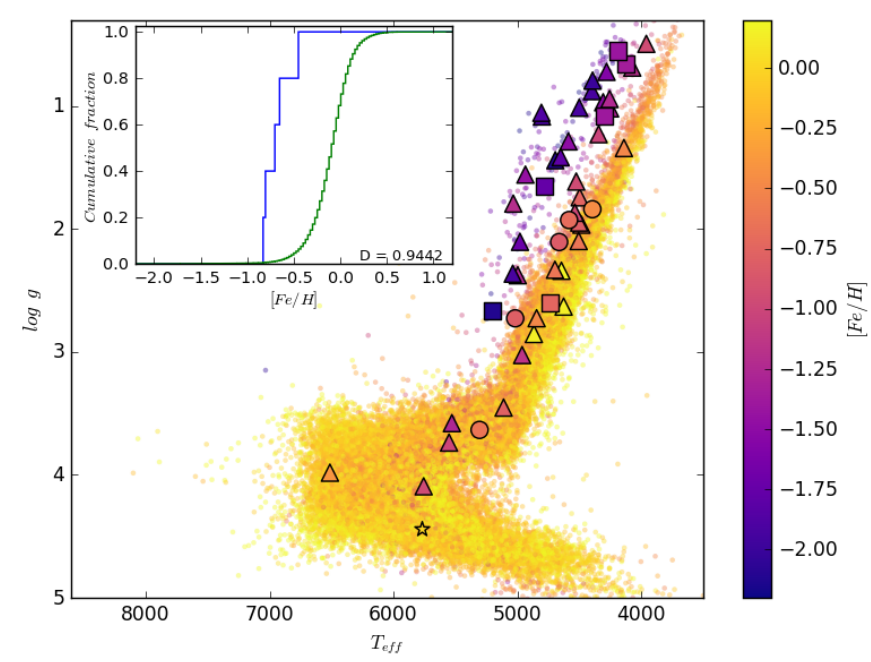

Figure 3. Surface gravity versus effective temperature for all stars in the master catalogue with spectroscopic parameters. The 11 comoving stars with known metallicity are shown as circles (confirmed members) or squares (possibles), the remainder as triangles. The location of the Sun is shown as a star for reference. The inset shows the normalised cumulative histogram of $[\mathrm{Fe} / \mathrm{H}]$ for the 5 secure comoving stars (in blue) and for the thin disk (in green). The $D$ value for the KS-test indicates that the two distributions are very different.

This cut leave us with 56 stars moving on predominantly eccentric orbits (41 are from the RAVE-on crossmatch, 15 from the other crossmatches). Fig. 1 shows the spatial distribution of all these stars, colour-coded according to metallicity (if known), with arrows representing their velocity vectors. The existence of a comoving cohort of stars at $(X, Y, Z) \approx(9.0,-1.0,-0.6) \mathrm{kpc}$ is evident. This is confirmed by a visual inspection of histograms of velocity components resolved with respect to the cylindrical polar coordinate $\left(v_{R}, v_{\phi}, v_{z}\right)$, which betrays clear peaks corresponding to the comoving clump. To formalize their extraction, we perform $2.5 \sigma$ clipping based on $v_{R}, v_{\phi}, v_{z}$ and orientation angle $\Psi$, individually. Here, $\Psi=\arctan (-V / U)$, where $U$ and $V$ are the Galactocentric Cartesian velocity components along the $X$ and $Y$ directions. So, $\Psi$ is the angle of star's motion in Galactic plane. Then, only those stars falling within the range of the median $\pm 2.5 \sigma$ (measured after the clipping) are retained. This process is repeated until no further stars are rejected. This leaves a set of 14 stars possibly belonging to a comoving clump with strongly radial orbits. They are shown as circles or squares in the left panel of Fig. 1, color-coded accord- ing to metallicity. All these stars lie in the TGAS and RAVE-on crossmatch.

For this set of 14 stars, we measured the median and $\sigma$ (standard deviation) in each $(U, V, W)$ component. To ensure that no possible members have been missed, we returned to the original sample of 73268 and searched for all stars in the $(U, V, W)$ velocity box bounded by the median $\pm 2 \sigma$ in each velocity component. This covers a comparable but wider range then the 'minimum to maximum' range in each velocity component of the 14 initial stars, so it is wide enough to contain our initial sample and any others that may have some comparable motion. However, no further stars are identified as possible members.

Astraatmadja \& Bailer-Jones (2016) used a prior suitable for nearby disk stars and they caution that their distances may be underestimates when objects lie beyond $2 \mathrm{kpc}$ from the Sun. Our comoving candidates have heliocentric distance $\approx 1.8 \mathrm{kpc}$, so it is prudent to cross-check our candidates with another different distance estimator. There are 8 RAVE-on stars among our candidates that satisfy recommendations advocated by Casey et al. (2016), namely they have teff_sparv $<8000 \mathrm{~K}$, the RAVE spectral parameters $c_{1}, c_{2}, c_{3}$ reported as ' $\mathrm{n}$ ' or normal, and a reduced $\chi^{2}<3$ as recorded by 'the Cannon' pipeline. For these stars, we can use the technique pioneered by Burnett \& Binney (2010) to compute the spectrophotometric distance distribution folded with the TGAS parallax distribution for each star. Specifically, we use the PARSEC isochrones (Bressan et al. 2012) and the extinction map of Green et al. (2015) to obtain the probability distribution function of the distance given the spectroscopic parameters $\left(T_{\text {eff }}, \log g,[\mathrm{Fe} / \mathrm{H}]\right)$, apparent 2MASS magnitudes $\left(J, H\right.$ and $\left.K_{s}\right)$ and the TGAS parallaxes along with their associated uncertainties (or covariances where available). We adopt an identical prior to that used in Binney et al. (2014). The right panel of Fig. 1 shows the new view of the comoving candidates, Of the 8 stars with spectrophotometric distances, 5 have a common velocity and metallicity. We regard these as confirmed members and they are shown as circles. Their RAVE identifiers, as well as right ascensions and declination, are given in Table 1. There are 3 remaining stars, 2 of which are no longer comoving, and 1 of which is comoving but has a discrepant metallicity. We regard these as merely possible members, pending confirmation of the distance or metallicity, and they are shown as squares. In other words, we have 5 confirmed members and 9 possible members, including the stars without spectrophotometric distances.

In the panels of Fig. 2, the circles and squares show our selected clump or co-moving group, while the triangles are the rejected stars (but still possessing $\epsilon_{\mathrm{RV}}<10 \mathrm{kms}^{-1}, \epsilon_{\mathrm{Vtot}}<25 \mathrm{kms}^{-1}$, 
relative velocity error $<10 \%$ and $J_{R}>800 \mathrm{kms}^{-1} \mathrm{kpc}$ ). The stars are colour-coded according to their radial and vertical velocities, so a coherent group stands out as a clump of objects with similar colouring. The significance of the comoving group above the background level can be measured in Galactocentric $(X, Y),(X, Z)$ and $(Y, Z)$ planes. The number of stars (with $\epsilon_{\mathrm{RV}}<10 \mathrm{kms}^{-1}, \epsilon_{\mathrm{V} \text { tot }}<25$ $\mathrm{kms}^{-1}$, relative velocity error $<10 \%$ and $J_{R}>800 \mathrm{kms}^{-1} \mathrm{kpc}$ ) in the comoving group region (drawn as an aperture that encloses the group) was compared with the number of stars in other regions (representing the background level) across the plane. The significance of the comoving group above the background level is $3.2 \sigma$, $7.2 \sigma$ and $4.8 \sigma$ for each Galactocentric $(X, Y),(X, Z)$ and $(Y, Z)$ planes respectively.

The comoving sample of 14 stars has median position $(X, Y, Z)=(9.00,-0.94,-0.62) \mathrm{kpc}$ and velocity $\left(v_{R}, v_{\phi}, v_{z}\right)=$ $(167.33,0.86,-94.85) \mathrm{kms}^{-1}$ in the Galactic rest frame. By integrating orbits in potential MWPotential2014 from Bovy (2015), we obtain a median apocentric distance of $\sim 13 \mathrm{kpc}$ and pericentric distance of $\sim 0.4 \mathrm{kpc}$ for stars in the clump. The high eccentricity (median $e=0.940$ ) and low apocentric distances suggest that the clump may be the relic of an object that fell into the halo long ago and whose apocentric distance has been reduced by dynamical friction over a number of pericentric passages. These values are recorded in Table 2. where we also give the corresponding figures if the sample is restricted to just the 5 stars with confirmed membership.

Fig. 3 shows the spectroscopic properties of the stars. They are all metal-poor giants with median $[\mathrm{Fe} / \mathrm{H}]=-0.83$. By conducting a Kolmogorov-Smirnov test, we can verify that the metallicities are not consistent with being drawn from the thin disk $(D=0.944)$. This is illustrated in the inset to the figure. The medians and median absolute deviations in the spectroscopic quantities are also noted in Table 2. As the stars in the comoving clump are exclusively drawn from the RAVE survey, an immediate concern is that the footprint of the survey may affect the results. The RAVE footprint and the comoving clump are shown in Galactic coordinates in Fig. 4. The positions of the 14 stars are marked in red. The distribution of circles (confirmed members) is suggestive of a stream moving from upper left to lower right. The three squares (possibles) at $\left(\ell \approx 245^{\circ}, b \approx-40^{\circ}\right)$ then lie $\sim 300 \mathrm{pc}$ off the stream. This also hints that these three stars are less likely to be members of the structure. However, this is not conclusive as it is conceivable that a globular cluster stream could broaden over time. Note that the substructure does extend towards the edge of the RAVE footprint, and so it is conceivable that it may be larger than is apparent from the TGAS and RAVE cross-match.

\section{DISCUSSION AND CONCLUSIONS}

Using crossmatches of the Tycho Gaia Astrometric Solution (TGAS) with the Radial Velocity Experiment (RAVE-on), we have extracted a sample of 14 stars with unusual kinematics. The stars are all moving on strongly radial orbits and are clumped at $(X, Y, Z) \approx(9.0,-1.0,-0.6) \mathrm{kpc}$. The median eccentricity of the stars is 0.940 .

We checked that this comoving group is resilient against distance errors. For 8 out of the 14 stars, it is possible to calculate spectrophotometric distances using the methods of Burnett \& Binney (2010). 6 of the stars still show a comoving trend. These 6 stars also have a much tighter $[\mathrm{Fe} / \mathrm{H}]$ distribution $\sim-0.7$ with one exception $([\mathrm{Fe} / \mathrm{H}]=-2.12)$. The median absolute deviation of $[\mathrm{Fe} / \mathrm{H}]$

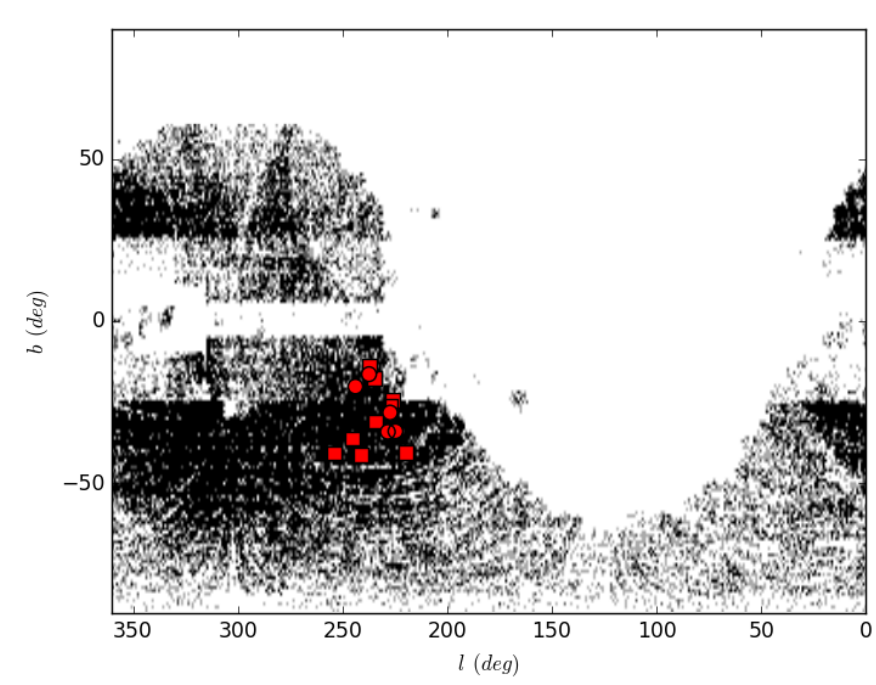

Figure 4. The RAVE footprint in Galactic coordinates. The 14 comoving clump stars are shown as circles (confirmed members) or squares (possibles). As the edge of the footprint abuts the clump, there may be an extension of the substructure that is currently missing.

is 0.10 . So, the motion, spatial location and metallicity of at least 5 stars are well confined in both distance estimates, suggesting that they are comoving and have a common origin.

The simplest interpretation of this coherently moving substructure is that it is the residue of a long past accretion event. The small metallicity spread in the 5 secure members suggests that the progenitor was more likely to be a globular cluster than a dwarf galaxy. The comoving group may be identified with the nucleus of this disintegrating body. Naturally, we would expect tidal streams also to be present, but in an old accretion event, the surface density of stream material mey be very low. Although some streams can be caused by resonance effects, such as the Hercules Stream (Dehnen 2000) and some can be caused by interactions with perturbers, such as the Aquarius Stream (Casey et al. 2014), neither option seems likely here. Resonance effects usually give structures of modest eccentricity, as nearly circular orbits can usually couple most easily to the perturbation.

The large globular cluster $\omega$ Centauri has long been thought to be the remnant nucleus of an accreted dwarf galaxy (Freeman 1993), as it shows evidence of multiple stellar populations with spreads in the age and metallicity (e.g. Villanova et al. 2007). If our comoving group is associated with $\omega$ Centauri, for example, by sharing a common progenitor, some clues may be found from stellar parameters or dynamics. RAVE DR4 (Kordopatis et al. 2013) provides an estimate of the age for 14 comoving group members. The median age is $9.8 \mathrm{Gyr}$ with standard deviation of $1.2 \mathrm{Gyr}$. The age-metallicity relation for $\omega$ Centauri presented by Villanova et al. (2014) shows some stars with metallicity and the age comparable to our comoving group stars, even though this estimated age is a relative age. Yet, the orbit of the comoving group appears to be highly eccentric with $v_{\phi}$ close to zero. It is ambiguous as to whether the group is prograde or retrograde, while $\omega$ Centauri clearly has a less eccentric and retrograde orbit (Majewski et al. 2000). In addition, the estimated values of energy and angular momentum of the comoving group stars do not show a clear link with $\omega$ Centauri, suggesting that this comoving group is very unlikely to be assocaited with it. 
Although there have already been searches for halo overdensities in the TGAS-RAVE cross-match by Helmi et al. (2017, herafter H17), the substructure identified in this paper appears to be new. It does not correspond to any of the overdensities labelled VelHel-1 to 9 in H17. As the underlying Galactic potential is different between the two papers, the estimated values of energy $E$ of the stars are different. $\mathrm{H} 17$ either convert parallaxes to distances via the reciprocal (70 \% of their sample) or use the RAVE distances (30\%). These are not the same as our spectrophotometric distances, so the azimuthal actions $J_{\phi}$ of the stars are also different. This affects the integrity of the substructures identified in H17, which we find to be indistinct and smeared out. As judged by the Tycho-IDs, there are only 4 stars in our substructure that overlap with H17, two in the VelHel-1 and 2 in VelHel-8. The claimed enhancements in H17 therefore do not appear to be related to our substructure, which is a coherent entity in configuration and velocity space.

\section{ACKNOWLEDGEMENTS}

GCM thanks Boustany Foundation, Cambridge Commonwealth, European \& International Trust and Issac Newton Studentship for their support on his work. SK thanks the United Kingdom Science and Technology Council (STFC) for the award of Ernest Rutherford fellowship (grant number ST/N004493/1). We thank the referee (Carl Grillmair) and Paul McMillan for helpful comments on the manuscript. This work has made use of data from the European Space Agency (ESA) mission Gaia (https://www.cosmos.esa.int/gaia), processed by the Gaia Data Processing and Analysis Consortium (DPAC, https://www.cosmos.esa.int/web/gaia/dpac/consortium). Funding for the DPAC has been provided by national institutions, in particular the institutions participating in the Gaia Multilateral Agreement.

\section{REFERENCES}

Anders, F., Chiappini, C., Santiago, B. X., et al. 2014, A\&A, 564, A115

Astraatmadja, T. L., \& Bailer-Jones, C. A. L. 2016, ApJ, 833, 119

Belokurov, V., Zucker, D. B., Evans, N. W., et al. 2006, ApJ, 642, L137

Binney J., 2010, MNRAS, 401, 2318

Binney J., et al., 2014, MNRAS, 437, 351

Bovy, J. 2015, ApJS, 216, 29

Bressan A., Marigo P., Girardi L., Salasnich B., Dal Cero C., Rubele S., Nanni A., 2012, MNRAS, 427, 127

Burnett, B., \& Binney, J. 2010, MNRAS, 407, 339

Casey, A. R., Keller, S. C., Alves-Brito, A., et al. 2014, MNRAS, 443, 828

Casey, A. R., Hawkins, K., Hogg, D. W., et al. 2016, arXiv:1609.02914

Dehnen, W. 2000, AJ, 119, 800

Freeman, K. C. 1993, in Smith, G., Brodie, J., eds, ASP Conf. Ser. Vol. 48, The Globular Clusters-galaxy Connection. Astron. Soc. Pac., San Francisco, p.608

Gaia Collaboration, Prusti, T., de Bruijne, J. H. J., et al. 2016, A\&A, 595, A1

Gaia Collaboration, Brown, A. G. A., Vallenari, A., et al. 2016, A\&A, 595, A2

Green, G. M., Schlafly, E. F., Finkbeiner, D. P., et al. 2015, ApJ, 810, 25

Grillmair, C. J. 2009, ApJ, 693, 1118

Helmi, A., White, S. D. M., de Zeeuw, P. T., \& Zhao, H. 1999, Nature, 402, 53

Helmi, A., Veljanoski, J., Breddels, M. A., Tian, H., \& Sales, L. V. 2017, A\&A, 598, A58

Høg, E., Fabricius, C., Makarov, V. V., et al. 2000, A\&A, 355, L27

Ibata, R. A., Gilmore, G., \& Irwin, M. J. 1994, Nature, 370, 194
Kunder, A., Kordopatis, G., Steinmetz, M., et al. 2017, AJ, 153, 75

Kordopatis, G., Gilmore, G., Steinmetz, M., et al. 2013, AJ, 146, 134

Luo, A.-L., Zhao, Y.-H., Zhao, G., et al. 2015, Research in Astronomy and Astrophysics, 15, 1095

Majewski, S. R., Patterson, R. J., Dinescu, D. I., et al. 2000, in Noels, A., Magain. P., Caro, D., Jehin, E., Parmentier, G., Thoul, A. A., eds, Proc. 35th Liege Int. Astrophys. Colloq., The Galactic Halo : From Globular Cluster to Field Stars. Institut d'Astrophysique et de Geophysique, Liege, Belgium, p.619

Newberg H. J., Carlin J. L. 2015, Tidal Streams in the Local Group and Beyond, Springer, New York

Smith, M. C., Evans, N. W., Belokurov, V., et al. 2009, MNRAS, 399, 1223

Villanova, S.,Piotto, G., King, I. R., et al. 2007, ApJ, 663, 296

Villanova, S., Geisler, D., Gratton, R. G., \& Cassisi, S. 2014, ApJ, 791, 107 